\title{
Conference: Morphological, Natural, Analog and Other Unconventional Forms of Computing for Cognition and Intelligence, Berkeley $2019^{+}$
}

\author{
Marcin J. Schroeder ${ }^{1, *}$ and Gordana Dodig-Crnkovic ${ }^{2}$ \\ 1 Global Learning Center, Tohoku University, Sendai, Miyagi Prefecture 980-8576, Japan \\ 2 Department of Computer Science and Engineering, Chalmers University of Technology, \\ 40482 Gothenburg, Sweden; gordana.dodig-crnkovic@chalmers.se \\ * Correspondence: schroeder.marcin.e4@tohoku.ac.jp; Tel.: +81-22-795-3246 \\ + Conference Morphological, Natural, Analog and Other Unconventional Forms of Computing for \\ Cognition and Intelligence (MORCOM), Berkeley, CA, USA, 2-6 June 2019.
}

Published: 7 May 2020

\begin{abstract}
The leading theme of the 2019 Summit of International Society for the Study of Information held 2-6 June 2019 at The University of California at Berkeley was the question "Where is the I in AI and the meaning of Information?" The question addresses one of the central issues not only for scientific research and philosophical reflection, but also for technological, economic, and social practice. The Conference "Morphological, Natural, Analog, and Other Unconventional Forms of Computing for Cognition and Intelligence" (MORCOM 2019) was focused on this theme from the perspective of unconventional forms of computing. The present paper, written by the organizers of the conference, reports the objectives of MORCOM 2019 and provides an overview of the contributions.
\end{abstract}

Keywords: artificial intelligence; intelligence; unconventional computing; morphological computing; natural computing; meaning of information

1. Conference "Morphological, Natural, Analog, and Other Unconventional Forms of Computing for Cognition and Intelligence" (MORCOM 2019): Objectives

Thus, intelligence and meaning, sources of hot controversies in the past are reincarnated now in the questions about AI. The questions regarding the modern information processing technology in performing tasks traditionally considered as exclusively human, or even considered as defining human beings, such as thinking, intelligence, consciousness, or goal-oriented agency, are essentially the same questions as those asked by natural philosophers through the ages. We have now more powerful intellectual and technological tools in searching for answers, but the existing pervasive and convenient tool-kit brings also a danger of following the old habits of thinking.

It is necessary to reconsider and reexamine even most fundamental concepts, such as computing or cognition and intelligence. As artificial intelligence is being constructed with the human intelligence as the first goal to achieve, the questions related to the nature of human intelligence have central importance. Constructing "intelligent artefacts" and comparing their features with human intelligence is a recursive process where learning goes two ways, from nature to artefact and back, as Rozenberg and Kari argue in "The Many Facets of Natural Computing" [1].

"AI Makes Philosophy Honest" argued Daniel Dennett in his talk at the I-CAP 2006 International Computers and Philosophy Conference in Laval, France. Instead of pure speculative thinking, AI provides us with a laboratory for testing hypotheses about intelligence and cognition. 
There are examples of novel studies, for instance, of morphological computing and embodied cognition, that succeed in escaping the inertia of thinking habits and question conventional theoretical and practical models.

The Conference MORCOM 2019 was not the first gathering of researchers and philosophers devoted to the discussion of the role of unconventional forms of computing in understanding natural and modelling artificial intelligence. Unconventional computing was proposed as a solution for overcoming the limitations which in the conventional form of computation were sources of objections to the intelligence of AI systems. There was a similar event on this subject at the IS4SI Summit 2017 in Gothenburg, Symposium on Morphological Computing and Cognitive Agency, [2]. It was followed by the event in 2018, Morphological and Embodied Computing Symposium on theory and applications [3], attended by philosophers, theoreticians and practitioners from several related fields

Each of the past gatherings was a step forward to better understanding of the alternative ways of computing in the context of their potential contributions to understanding of natural cognition and intelligence as well as to the design of cognitive intelligent artefacts.

MORCOM 2019 brought together perspectives on morphological, physical, natural, analog and embodied cognitive computation and other forms of unconventional conceptualization of computing, cognition and intelligence. We, as organizers, encouraged open and constructive debate on the perceived differences in the various perspectives on constructivist and computationalist accounts of the dynamics of information in its natural and artefactual realizations. Contributed presentations gave very diverse perspectives on the relevant subjects.

It is difficult to report or summarize multiple, more or less formal, discussions which followed the presentations and also were carried out at subsequent informal events. In the following, a very brief account of the subjects of presented works can give a basic idea about the MORCOM Conference and its contributions.

\section{Conference "Morphological, Natural, Analog, and Other Unconventional Forms of Computing for Cognition and Intelligence" (MORCOM 2019): Contributions}

Todd Hylton's keynote lecture “Thermodynamic Computing: An Intellectual and Technological Frontier" of MORCOM 2019 and of the entire Summit 2019 addressed one of the crucial differences between the structure of the brain and the structure of artificial neural networks. While traditional embodiment of artificial neural networks has static and passive architecture where the network learning is entirely controlled by the iterated external input, we know that the neural network in the human brain is a self-organized system in which this self-organization is an active foundation of learning.

Hylton's approach is based on a self-organized artificial neural network utilizing self-organization of minuscule conductor particles floating in viscous insulating liquid under the influence of an external electric field. It is critical for this process that the external electric field is not determining the structure developed by the particles, but it is rather a stimulating agent for self-organization. This approach is a revolutionary development towards future technologies utilizing self-organized systems for intelligent technologies, based on mechanisms similar to those in living beings.

There were two submissions to MORCOM 2019 in the broadly understood direction of the study of embodiment of unconventional computing which, unfortunately, could not be presented in their entire extent at the conference due to logistic obstacles preventing attendance of the contributors at the summit. Yet the works, summarized in extended and informative abstracts, generated interest and discussion among the participants. One of them, of the authorship of Ricardo Q. Figueroa, Genaro J. Martinez, Andrew Adamatzky and Luz N. Oliva-Moreno, entitled "Robots Simulating Turing Machines" addressed one of the classical subjects of computer science considering machines simulating machines, but in the context of unconventional computing and with the focus on embodiment. 
The other work contributing to the conference with the subject for discussion rather than reporting of the results entitled "Propagation of Patterns in Non-linear Media as a Paradigm of Unconventional Computers" was authored by Genaro J. Martinez, Andrew Adamatzky, Ricardo Q. Figueroa and Dmitr A. Zaitsev. Cellular automata are classic models to design unconventional computing in several ways. In their work, the authors adopt an analogy between precipitating chemical, physical or biological media and semi-totalistic binary two-dimensional cellular automata. In this analogy, the patterns, originating from different sources of perturbations, propagating in a precipitating chemical, physical or biological medium, compete for the space. They sub-divide the medium onto the regions unique for an initial configuration of disturbances. This sub-division can be expressed in terms of computation. The work of Martinez et al. demonstrates how to implement basic logic and arithmetical operations (its computability) by patterns propagating in geometrically constrained cellular automata medium. This opens the way to design and to implement computation in a very wide range of physical and chemical media.

The Skype presentation by Hector Zenil "Towards Demystifying Shannon Entropy, Lossless Compression, and Approaches to Statistical Machine Learning" presents a critical analysis of the current approaches of machine and deep learning based on traditional statistics and information theory, showing that they fail to capture fundamental properties of our world and are ill-equipped to deal with high-level functions such as inference, abstraction, and understanding.

In contrast, Zenil explored recent attempts to combine symbolic and sub-symbolic/differentiable computation in a form of unconventional hybrid computation that is more powerful and may eventually display and grasp these higher level elements of human intelligence. In particular, he introduced the field of Algorithmic Information Dynamics and that of Algorithmic Machine Intelligence based on the theories of computability and algorithmic probability, and demonstrated how these approaches promise to shed light on the weaknesses of current AI (especially Deep Learning, which also Joshua Bengio agrees with) and how to attempt to circumvent some of their limitations.

Vincent C. Müller, in his Skype presentation "Morphological Computation and the Discussion about Whether Computation Involves Meaningful Symbols" referred to the discussion about morphological computation and about whether computation involves meaningful symbols, rather than merely syntactic operations. He described his position that computation, as it is traditionally understood, is essentially syntactic algorithmic processing (as the Church-Turing thesis claimed) done by humans with machines. However, there are other, unconventional and very fruitful and plausible notions of computing, for instance, those engaging morphology. Thus, Müller stated that the question about the possibility of computing involving meaning requires scrutiny. What kind of question is this? Do we expect a discovery to find out the truth, or can we slice the world in several plausible ways? Is this essentially the same question as about the philosophical positions of realism and anti-realism applied to computing?

Rao Mikkilineni presented his work with Mark Burgin and Eugene Eberbach "Processing Information in the Clouds" which expressed the view that the way to Higher-Order AI, i.e., to the design of actual intelligent artefacts, leads in the direction of cloud computing as a means to overcome limitations of localization of computational process in the system embodying computation.

In the presentation of his work with Mark Burgin "Structural Machines as Unconventional Knowledge Processors" Rao Mikkilineni involved the concept of knowledge and knowledge processing to describe some new form of unconventional computing understood as knowledge processing, distinguished from information processing. Mikkilineni and Burgin used analogy to demonstrate their view of knowledge as a concept of higher complexity than information: knowledge contains information as matter contains energy. Since knowledge may require very complex structures which are not necessarily linear, while conventional computing machines and automata are typically sequential processing of information, processing of knowledge requires higher forms of computing.

Rao Mikkilineni presented his individual work on a similar subject "Information Processing, Information Networking, Cognitive Apparatuses and Sentient Software Systems" in which he 
presented a new information processing architecture that enables "digital genes" and "digital neurons" with cognizing agent architecture to design and implement sentient, resilient and intelligent systems in the digital world. His approach was motivated by the recognition of the fact that computing processes, message communication networks and cognitive apparatuses are the building blocks of living sentient beings. Genes and neural networks provide complementary information processing models that enable execution of mechanisms dealing with "life" using physical, chemical and biological processes. Cognizing agent architecture (mind) provides the orchestration of body and the brain to manage the "life" processes to deal with fluctuations and maintain survival and sustenance.

Mikkilineni's presentations belonged to the more theoretical direction of the MORCOM Conference. Another highly theoretical work "Processing Information by Symmetric Inductive Machines" presented by Mark Burgin, was devoted to analysis of the model of computation introduced in 2013 by Marcin J. Schroeder as Symmetric Turing Machines or S-machines. In this model, one-way action of the head (processor) on the tape (memory) is replaced by interaction between two essentially equivalent components of the machine.

Automata that perform transformations with their programs, such as reflexive Turing machines, were explored by Burgin in 1992. It was proved that these machines have the same computing power as Turing machines but could be much more efficient.

Using a technique similar to the one employed in the past, it is possible to prove that functioning of a symmetric Turing machine can be simulated by a conventional Turing machine with two tapes and two heads. It means that symmetric Turing machines have the same computing power as Turing machines. At the same time, it is also possible to prove that symmetric Turing machines can be much more efficient than Turing machines.

To achieve higher computing power, Burgin introduced and initiated study of inductive symmetric machines, which further develop the structure and possibilities of inductive Turing machines allowing to model natural computations in various situations.

In the discussion after the presentation, Schroeder pointed out that the equivalence of the symmetric Turing machine with two heads and two tapes assumes that the dynamics of interaction is described by a computable function, which his original model did not assume. Thus, consideration of the symmetric Turing machine with non-computable dynamics of interaction re-opens the question about its computational power. Schroeder admitted that, at present, his example of such a model is rather artificial, but it demonstrates a possible direction of further research alternative to Burgin's inductive symmetric machine.

Lorenzo Magnani, in his presentation "Disseminated Computation, Cognitive Domestication of New Ignorant Substrates, and Overcomputationalization" presented "eco-cognitive computationalism" understood as a study of computation in context, following some of the main tenets advanced by the recent cognitive science views on embodied, situated, and distributed cognition.

In this eco-cognitive perspective, Magnani analyzed the recent attention in computer science devoted to the importance of cognitive domestication of new substrates, such as in the case of morphological computation. This new perspective shows how the computational domestication of ignorant substrates can originate new unconventional cognitive embodiments, which expand the processes of computationalization already occurring in our societies.

Magnani also introduced and discussed the concept of overcomputationalism, as intertwined with the traditional concepts of pancognitivism, paniformationalism, and pancomputationalism, seeing them in a more naturalized intellectual disposition, appropriate to the aim of bypassing ontological or metaphysical overstatements. 
What he called overcomputationalization refers to the presence of too many entities and artefacts that carry computational tasks and powers. Overcomputationalization (1) often promotes a plenty of possible unresolvable disorganizational consequences, and (2) tends to favor philosophical reflections that depict an oversimplified vision of the world. Moreover, it tends to generate too many cognitive constraints and limitations, which lead to a weakening of human creative (abductive) cognitive activities, as he has illustrated in the last chapter of his recent book The Abductive Structure of Scientific Creativity (2017), and, because of the excess of redundant cognitive/informational features attributed to entities (features often exogenous to the original functions of them), it tends to prevent human intellectual freedom to benefit from that cognitive simplification that is characteristic of the absence of informational overloads.

A presentation by Gordana Dodig-Crnkovic "Morphological, Natural, Analog and Other Unconventional Forms of Computing for Cognition and Intelligence" provided a comprehensive review of the subject of MORCOM 2019; starting from the frequently asked fundamental questions: What is the relationship between Cognition and Intelligence? How does cognitive computing relate to AI? What is the difference between Natural, Analog and Morphological computing?

Dodig-Crnkovic demonstrated the need for establishing an appropriate conceptual framework in order to attempt providing answers, as at the moment, there is a huge variety of use of those terms that causes confusion. For this purpose, in her presentation, she elaborated on the taxonomy of computing originally developed in collaboration with Mark Burgin, and extended it by some recent work on cognition as information processing.

The thesis of Dodig-Crnkovic is that nonconventional (morphological) computation provides a good basis for both new and effective ways of computation that are energetically much more favorable than the ones we currently use, and for modelling of information processing in living organisms, thus intelligence and cognition involving meaning. It is equally well suited for modelling of natural as well as artificial intelligence.

Marcin J. Schroeder's presentation, "Intelligent Computing: Oxymoron?" was intended as the direct answer to the leading question of the Summit. Schroeder admitted that the denial of intelligence and capacity to understand meaning to computing machines requires prior definitions of the two concepts infamous for escaping all attempts of conceptualization. Thus, in the absence of such a commonly accepted conceptual framework, every claim attributing or denying intelligence and capacity to understand meaning to computing machines is more an invitation to further discussion than a final answer to the question. On the other hand, there are some characteristics of intelligence and meaning which are not sufficient, but necessary conditions for computing machines to be qualified as intelligent and as capable of understanding meaning.

For instance, it is difficult to expect that any entity (human or artificial) lacking the ability to reduce or eliminate complexity can be considered intelligent. Yet it can be demonstrated that a computing machine in the present model of computation is devoid of this capacity. This, of course, does not mean that computing systems with alternative forms of computation cannot be intelligent.

In conclusion, the Conference on Morphological, Natural, Analog and Other Unconventional Forms of Computing for Cognition and Intelligence at IS4SI summit in Berkeley 2019, provided plenty of stimulating insights into the field of unconventional and morphological computing pointing towards the answers to the central question of the Summit: agency and meaning of information in artefactually intelligent systems.

Funding: This research received no external funding.

Conflicts of Interest: The authors declare no conflict of interest. 


\section{References}

1. Rozenberg, G.; Kari, L. The Many Facets of Natural Computing. Available online: https://cacm.acm.org/ magazines/2008/10/511-the-many-facets-of-natural-computing/fulltext (accessed on 6 May 2020).

2. Symposium on Morphological Computing and Cognitive Agency. Available online: http://is4si-2017.org/ (accessed on 6 May 2020).

3. Morphological and Embodied Computing Symposium on theory and applications 2018. Available online: https://sites.google.com/view/morphologicalcomputing (accessed on 6 May 2020).

(C) 2020 by the authors. Licensee MDPI, Basel, Switzerland. This article is an open access article distributed under the terms and conditions of the Creative Commons Attribution (CC BY) license (http://creativecommons.org/licenses/by/4.0/). 